\title{
Diseño de agentes para recuperar información para el enriquecimiento de ontologías dirigidas a epidemiología: el caso de la tuberculosis
}

\author{
Joanna Alvarado-Uribe, Ari Yair Barrera-Animas, Miguel González-Mendoza, \\ Ma. de los Ángeles Junco-Rey \\ Instituto Tecnológico y de Estudios Superiores de Monterrey, \\ Edo. de México, México \\ joanna.1890@gmail.com, gnpliquid@gmail.com,mgonza@itesm.mx, \\ ajunco@itesm.mx
}

\begin{abstract}
Resumen. En este trabajo se presenta un Sistema Multiagente que busca información relevante en Sitios Web, que puede ser filtrada de tal manera que puede contribuir en el enriquecimiento de una ontología dirigida a la Tuberculosis en México. Esta ontología está basada, por un lado, en la estructura de la Base de Datos Relacional (BDR) del Sistema Nacional Mexicano de Vigilancia Epidemiológica (SINAVE), información estructurada; $y$, por otro lado, de un Sistema Multiagente (SMA) que recolecta información de la web en diferentes formas (información semiestructurada). Particularmente, los agentes recopilan información de distintas fuentes dinámicas: Facebook, Twitter y Blogs (páginas de Internet), generales y especializadas, que permiten la actualización de esta ontología. El análisis y diseño de los agentes se realiza utilizando la metodología GAIA y el protocolo de comunicación se lleva a cabo por medio del estándar FIPA-ACL; desarrollado en el entorno de programación JADE (Java) a través de NetBeans. Se realiza la conversión de la información relacional a semántica utilizando la herramienta D2R Server, para generar Ontologías por medio del método de mapeo directo de BDR.
\end{abstract}

Palabras clave: Sistema multiagente, ontología, GAIA, FIPA, JADE.

\section{Introducción}

Actualmente, cualquier búsqueda en la Web nos arroja todos los posibles resultados que estén relacionados con la petición que el usuario realizó, aunque no se asegura que todos los resultados sean únicamente los que se quieren conocer. Así, el usuario tiene que continuar su búsqueda manualmente en cada uno de los resultados, lo que consume más tiempo.

La incorporación del enfoque semántico en la Web nos asegura que todos los resultados de la búsqueda sean exactos [1], debido a que la información se encuentra mejor definida; esto es posible gracias a que la representación del conocimiento se realiza por medio de ontologías. Las ontologías permiten definir un vocabulario 
común que describe en un dominio específico, los conceptos que involucra y las relaciones entre ellos; por tal motivo, las ontologías se convierten en una pieza fundamental de las tecnologías orientadas a la Web Semántica [12].

Con base en el párrafo anterior, se plantea una forma para enriquecer una ontología de acuerdo con el tema que se requiera incorporar a cierto Sistema Computacional o en el caso más general, a la Web Semántica. En este trabajo se propone el enriquecimiento de una ontología acerca del tema de Tuberculosis en México. Esta ontología se compone de dos partes: la primera se basa en la estructura de una Base de Datos Relacional proporcionada por el Sistema Nacional Mexicano de Vigilancia Epidemiológica (SINAVE) dependiente del Sector Salud [3]; y la segunda, de información obtenida de páginas Web. Por lo que, se busca recopilar información de distintas fuentes estáticas y/o dinámicas, generales y especializadas; que permitan la actualización de esta ontología.

La búsqueda de información útil para el enriquecimiento de la ontología se realiza por medio de un Sistema Multiagente; dicho sistema se compone de 3 agentes: RSATFB (Recolector del Sistema Multiagente en Facebook), RSATTW (Recolector del Sistema Mutiagente en Twitter) y RSATBLOG (Recolector del Sistema Multiagente en los BLOGs). Estos agentes recopilan la información que los usuarios proporcionan en estos sitios y la analizan para determinar qué información podría ser relevante para la actualización de la ontología de Tuberculosis. Los términos que sean extraídos de estos sitios Web deben tener una alineación con al menos uno de los términos almacenados en la BDR de Tuberculosis. Una vez que analizan la información, la clasifican de acuerdo con el área a la que pertenezca y la almacenan en una Base de Datos Relacional que es mapeada manualmente para obtener la ontología actualizada.

El proceso de convertir la información relacional a semántica en este trabajo se realiza con la herramienta D2R Server, con la que se generan dos tipos de archivos: uno en formato RDF, que es el utilizado convencionalmente por la Web Semántica y; otro con extensión .ttl, que permite la explotación y el enriquecimiento de los datos semánticos utilizando SPARQL con un razonador denominado Snorql para hacer inferencia sobre los datos [3].

El uso de un Sistema Multiagente permite que la ontología vaya creciendo con información obtenida de diversas fuentes y con un grado mayor de confianza al considerar la definición de un agente "Los agentes son componentes de software activos y persistentes que perciben, razonan, actúan y se comunican" Huns \& Singh (1998) [2]. Estas características permiten a los agentes convertir una actividad humana a semiautomática.

A continuación, se describen los 5 apartados que conforman este artículo: Análisis y diseño, donde se realiza la descripción de la organización del sistema, los roles de los agentes, protocolos y relaciones; Prototipo, se describe el prototipo y la forma de usarlo; Experimentos y resultados, se describen las partes cubiertas hasta el momento de la propuesta; y Conclusiones. 


\section{Análisis y diseño}

El sistema se compone de 3 agentes: RSATFB (Recolector del Sistema Multiagente en Facebook), RSATTW (Recolector del Sistema Mutiagente en Twitter) y RSATBLOG (Recolector del Sistema Multiagente en los BLOGs) [7].

Estos agentes recopilan la información que los usuarios proporcionan en estos sitios, la analizan para determinar qué información es irrelevante o útil para la actualización de la ontología de Tuberculosis a partir de los términos que sean extraídos de estos sitios Web. Una vez que analizan la información, la clasifican de acuerdo con el área a la que pertenece, almacenando la información recolectada en una Base de Datos Relacional denominada SMAterminos (BDR SMAterminos). Posteriormente, los términos presentes en la BDR SMAterminos son comparados con los términos contenidos en la BDR de Tuberculosis (BDR), con el propósito de preservar exclusivamente términos nuevos. Finalizando con el mapeo manual de la BDR de Tuberculosis denominada "tuberculosis_uno", en el caso de haber clasificado términos nuevos, con la finalidad de obtener la ontología actualizada en formato RDF y .ttl para su explotación. Como se puede ver en la Fig. 1.

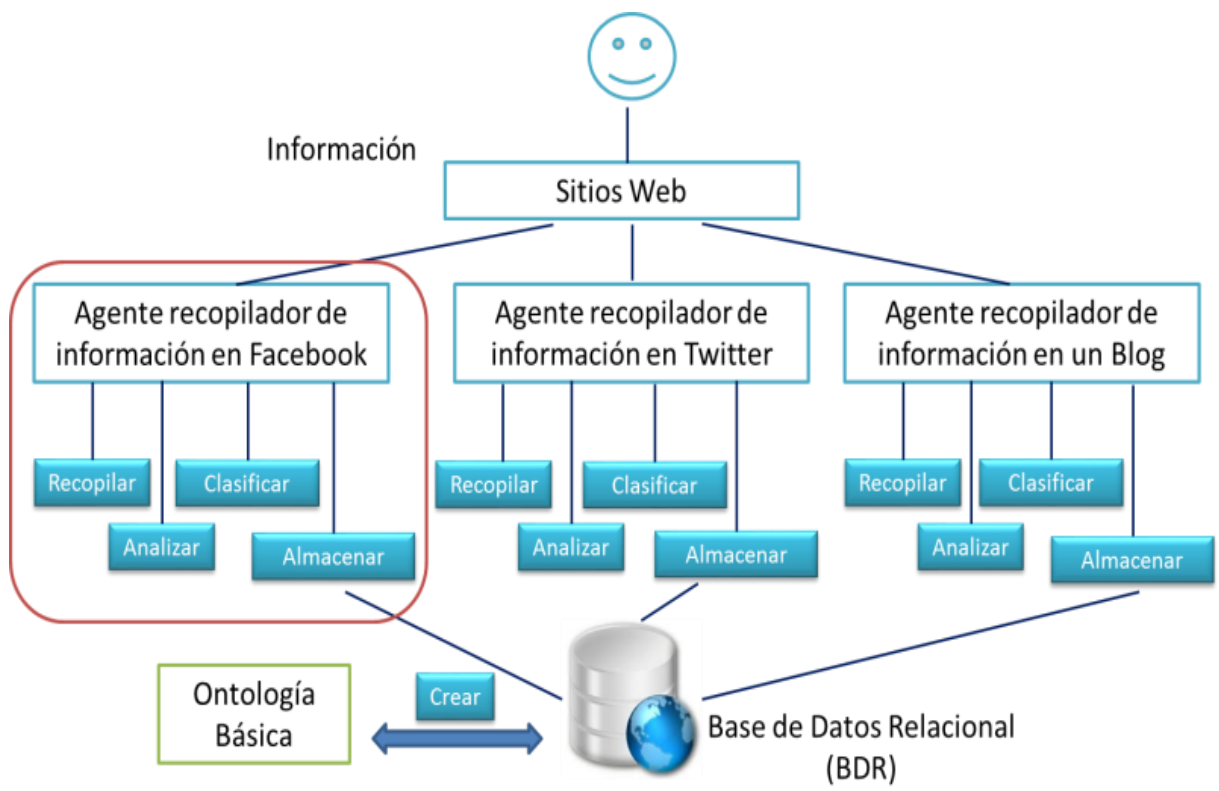

Fig. 1. Organización del sistema.

\subsection{Modelo del agente}

Se tienen tres modelos:

- Modelo del agente RSATFB (Recolector del Sistema Multiagente en Facebook), ver Fig. 2. 
- Modelo del agente RSATTW (Recolector del Sistema Multiagente en Twitter).

- Modelo del agente RSATBLOG (Recolector del Sistema Multiagente en Blogs).

Cabe mencionar que todos los agentes presentan el mismo modelo (actividades que ejecuta el agente).

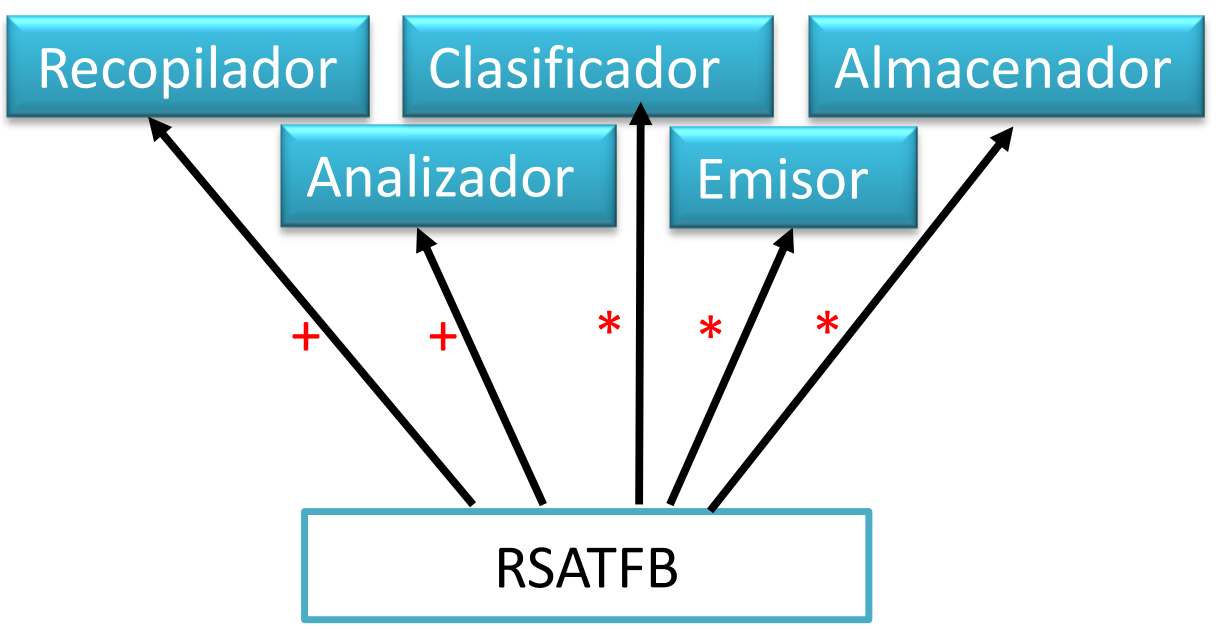

Fig. 2. Modelo del agente RSATFB.

\subsection{Modelo de roles (a nivel de análisis)}

Los agentes presentan, de manera específica y congruente a su función, un esquema de rol definido; en el que se establece el comportamiento del mismo a través del sistema. El esquema que se muestra en la Tabla 1 denota el rol para el agente RSATFB (Facebook), que funge como ejemplo demostrativo de los roles correspondientes a cada agente previsto.

Tabla 1. Esquema del rol del agente propuesto para Facebook.

\section{Esquema del Rol: RSATFB}

\section{Descripción:}

Este rol involucra recopilar información de la red social Facebook en las publicaciones de los usuarios para analizarla, identificar términos nuevos de acuerdo con la BDR SMAterminos, y la tabla correspondiente a su Sitio Web y almacenar dichos términos en la misma BDR, en el caso de que otro agente no lo haya identificado.

\section{Protocolos y Actividades:} Actividades: 
Diseño de agentes para recuperar información para el enriquecimiento de ontologías dirigidas ...

- Recopilar: consiste en extraer toda la información que los usuarios de Facebook publiquen en esta red social.

- Analizar: consiste en leer la BDR para determinar los términos que no se encuentran clasificados en la BDR SMAterminos. (Posteriormente, esta tabla será evaluada por un especialista en el área para introducir dichos términos a la Ontología de Tuberculosis).

- Clasificar: identifica e indica los términos nuevos y los que ya se han clasificado en su momento.

- Almacenar: consiste en guardar los nuevos términos en la BDR SMAterminos que se identifiquen.

\section{Protocolo:}

- Emitir: consiste en transmitir a los agentes que se identificó un nuevo término, los cuales revisan si en ese momento ya lo han identificado y si esto es verdadero, le responden con la performativa INFORM "Término clasificado"; de lo contrario, su respuesta es un "Término NO clasificado".

\section{Permiso:}

- Modificar la BDR SMAterminos.

\section{Responsabilidades:}

- De vida:

RSATFB $=(\text { Recopilar.Analizar })^{\mathrm{w}} \cdot(\text { Clasificar.Emitir })^{*} \mid$ Almacenar ${ }^{*}$

- De seguridad:

$>$ Nueva Publicación = "Sí".

$>$ Prioridad máxima al protocolo "Emitir".

\subsection{Modelo de interacción (a nivel de análisis)}

Se tiene únicamente un protocolo "Emitir", que tiene incluidas las actividades Analizar, Clasificar y Almacenar, siendo el mismo protocolo para todos los agentes contenidos en el sistema, presentando cada uno las modificaciones correspondientes en la implementación del protocolo "Emitir" con la finalidad de establecer la comunicación entre los otros agentes y recibir sus peticiones. En la Tabla 2 se muestra el esquema del protocolo de interacción "Emitir", que define la descripción de la interacción entre agentes cuyo propósito principal es la emisión (propagación y recepción) de todo nuevo término capturado por cada uno de los agentes.

Tabla 2. Esquema del protocolo Emitir.

\begin{tabular}{|l|c|}
\hline \multicolumn{2}{|c|}{ Propósito: Emitir término } \\
\hline \multicolumn{1}{|c|}{ Iniciador: RSAT } & $\begin{array}{c}\text { Respondedor: RSAT diferente a } \\
\text { Iniciador }\end{array}$ \\
\hline Descripción:
\end{tabular}


Transmitir a los agentes que se identificó un nuevo término, los cuales revisan si en ese momento ya lo han identificado y si esto es verdadero, le responden con la performativa INFORM "Término clasificado"; de lo contrario, su respuesta es un "Término NO clasificado" y lo introducen a la tabla termino_clasificado de acuerdo con el Sitio Web que exploran.

Entrada: Término Nuevo = "Termino NO clasificado".

Salida: Clasificación = "Término clasificado" || "Término NO clasificado".

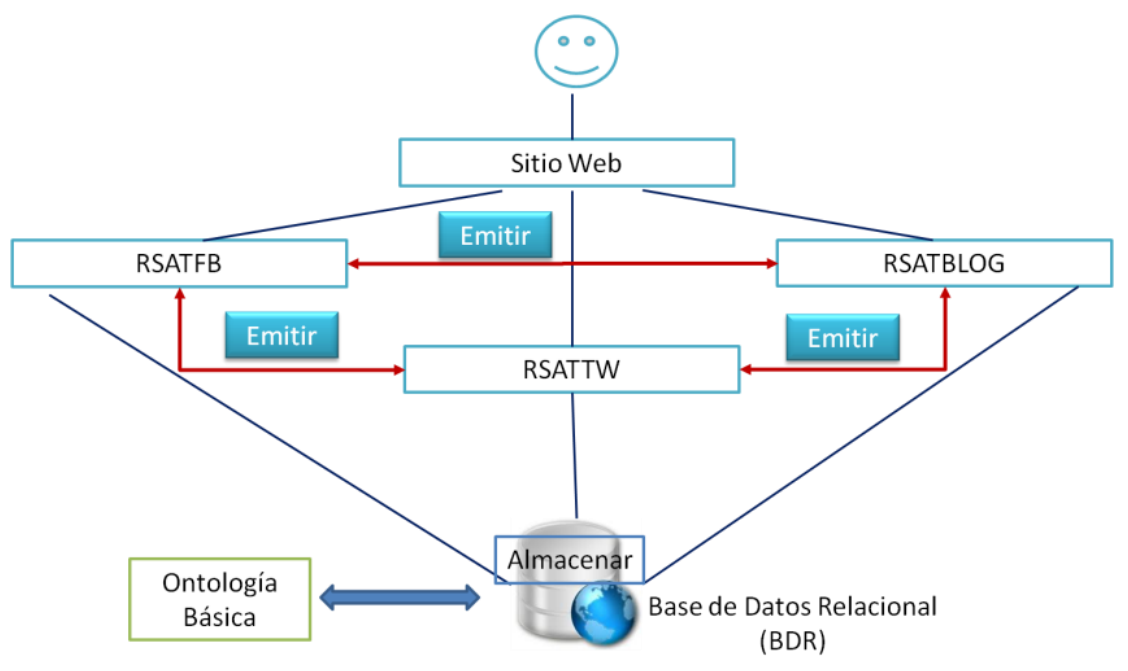

Fig. 3. Modelo de conocimiento.

\subsection{Conocimiento de los agentes}

Como conocimiento previo se tienen dos Bases de Datos Relacionales (BDR):

1) La primera BDR se denomina "tuberculosis_uno", esta Base de Datos contiene las tablas y los registros que se utilizan para construir la primera versión de la ontología. Se usa la ontología resultante como base para los agentes, con la finalidad de que todos los agentes presenten un vocabulario común y especializado en esta área.

2) La segunda BDR se denomina "SMAterminos", esta Base de Datos contiene tres tablas, una para los términos clasificados de Facebook (FB), otra para los términos clasificados de Twitter (TW) y la última para los Blogs (B). Estas tablas contienen los mismos campos: cve_termFB, nombre_termFB y desc_tablaFB; las siglas FB cambian respecto al Sitio Web de donde se obtienen los términos, ej. TW y B, donde

- cve_term es la clave identificadora del término recopilado del sitio Web especificado. 
Diseño de agentes para recuperar información para el enriquecimiento de ontologías dirigidas ...

- nombre_term contiene los nombres de los términos nuevos recopilados que cada agente va clasificando.

- desc_tabla contiene el nombre de la tabla correspondiente a la BDR "tuberculosis_uno" que el agente establece como una posible pertenencia del término que se clasificó.

Como conocimiento adquirido, se propone incrementar el vocabulario de la ontología con los términos nuevos que cada agente clasifica en la BDR SMAterminos, de acuerdo con las entradas de los Sitios Web y la validación de los términos que realizará un especialista en el área; que, hasta el momento, es el encargado de introducir cada término manualmente a la BDR "tuberculosis_uno".

\section{Prototipo}

El SMA propuesto está codificado en el entorno de programación JADE versión 4.3.0 (Java) a través de NetBeans IDE 7.0.1; se ejecutó en una Laptop con las siguientes características: Procesador AMD Quad - Core a $3.5 \mathrm{GHz}, 6 \mathrm{~GB}$ de memoria DDR3, 750 GB de Disco Duro, Sistema Operativo Windows 8 de 64 bits.

Al realizar la ejecución del Sistema Multiagente, hará que los tres Agentes (Agente Facebook $=$ RSATFB, Agente Twitter $=$ RSATTW, Agente Blog $=$ RSATBLOG) comiencen a recopilar información y de esta manera, se clasifiquen los términos nuevos en la BDR.

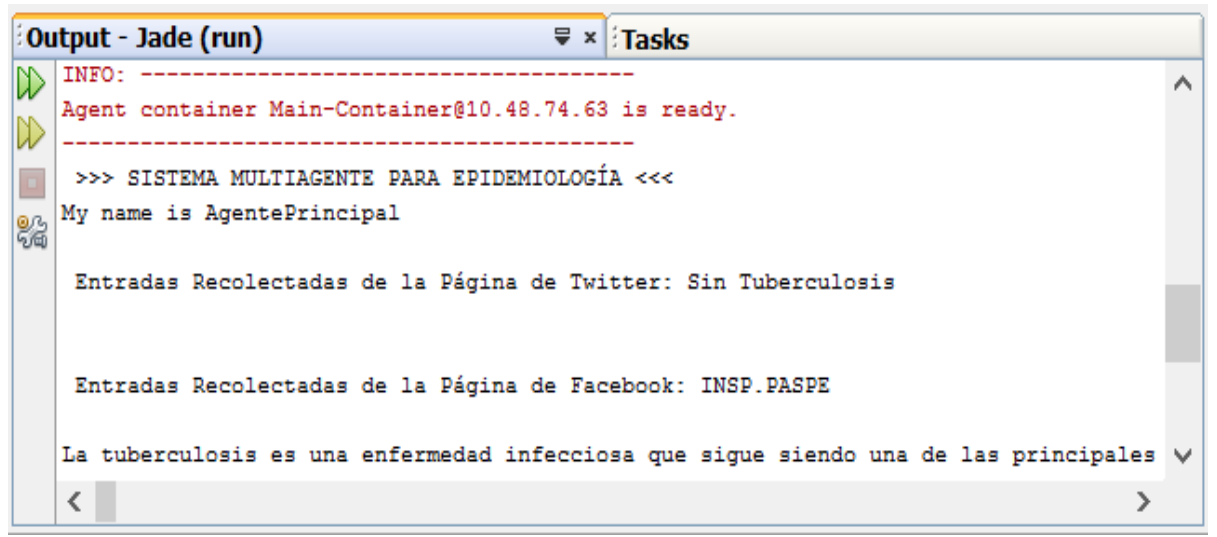

Fig. 4. Ejemplo de recopilación de información tras la ejecución de todos los agentes del sistema.

En la Fig. 4 se muestra un ejemplo del funcionamiento de los agentes al realizar la ejecución del Sistema Multiagente. Donde se puede observar que se realiza la recopilación de entradas a través de dos páginas Web: la primera es del agente recopilador de Twitter en el perfil denominado "Sin Tuberculosis" [13], del que no se tienen resultados para nuestra área de estudio al momento de la recopilación de datos; y la segunda es de la página de Facebook denominada "INSP.PASPE" [14] (entrada 
del agente recopilador de Facebook), en la que sí se visualiza una entrada para el área de Tuberculosis.

Es posible cambiar cada uno de los términos que "envía" o "busca" cada uno de los Agentes a través de sus respectivos Comportamientos. Para cambiar cada uno de los términos de cada Agente, se selecciona el Comportamiento EmitirBlog para el Agente Blog, el Comportamiento EmitirFacebook para el Agente Facebook y el Comportamiento EmitirTwitter para el Agente Twitter; una vez que se encuentra en el Agente que desea modificar, se localiza la variable "mensaje_a_enviar" y se modifica su valor por cualquier texto que se desee probar, ver Fig. 5.

Jade - NetBeans IDE 7.3

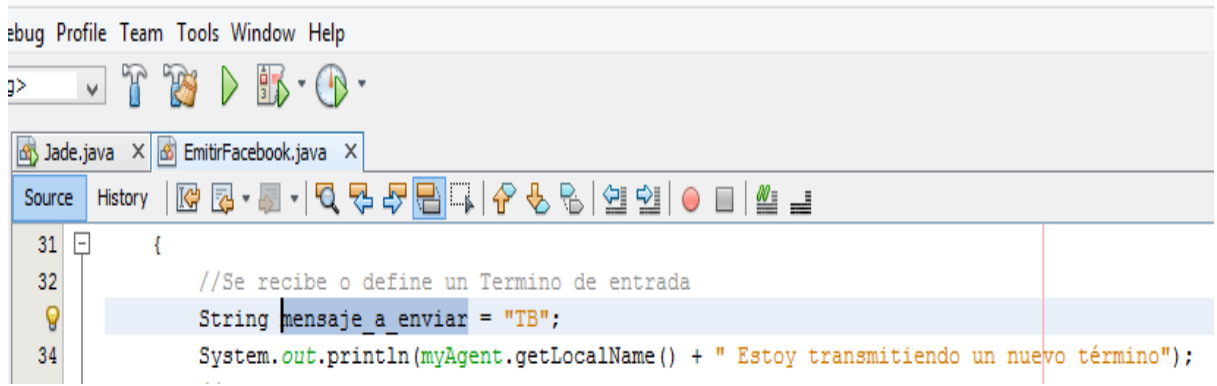

Fig. 5. Ejemplo de cambio de valor en la variable "mensaje_a_enviar" en EmitirFacebook.

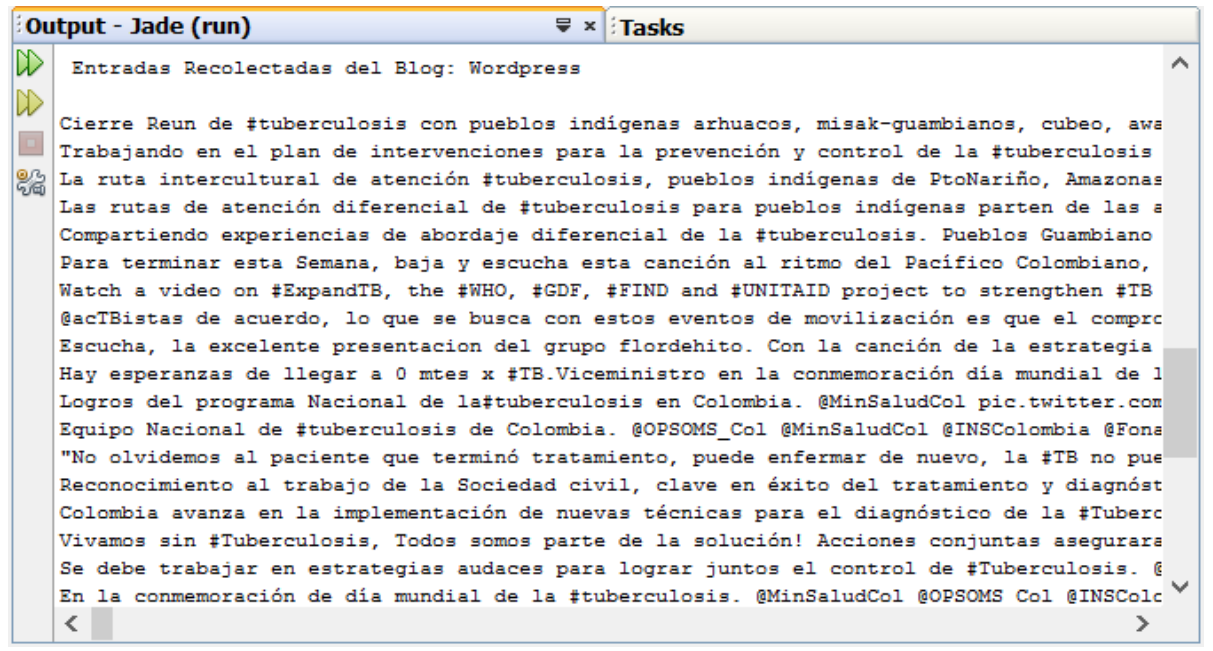

Fig. 6. Información obtenida del agente para BLOGs.

\section{Experimentos y resultados}

Los siguientes experimentos se llevaron a cabo.

- Extraer los términos de una publicación en un Sitio Web. La Fig. 6 muestra las entradas correspondientes a la página Wordpress [15] 
Diseño de agentes para recuperar información para el enriquecimiento de ontologías dirigidas ...

indicada en la sección de BLOGs (correspondiente al agente recopilador de BLOGs).

- Clasificar los términos. La Fig. 7 muestra las tablas que conforman a la BDR SMAterminos, que contiene los términos clasificados por los agentes en cada Sitio Web y los nombres de las tablas de la BDR "tuberculosis uno" a las que posiblemente pertenezcan los términos clasificados, éste se almacena en el campo desc_tabla. Estos dos valores son los que utiliza el especialista que valida los términos para verificar que efectivamente los agentes están realizando la clasificación correctamente $\mathrm{y}$, de esta manera, el especialista al verificar que la actividad Clasificar está ejecutándose satisfactoriamente, los agentes podrán directamente vaciar los términos clasificados en la BDR de Tuberculosis sin tener que esperar la validación del especialista en el área.

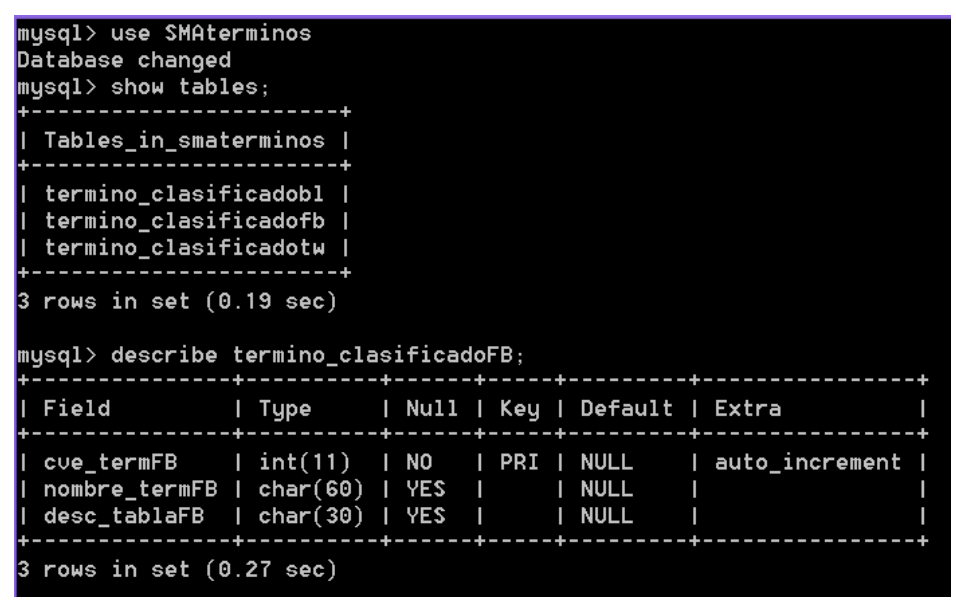

Fig. 7. Estructura de la BDR SMAterminos.

- Comunicación entre los agentes. Se realiza por medio del protocolo Emitir mediante performativas que son enviadas entre los agentes; las performativas utilizadas son REQUEST, INFORM y CONFIRM. Tal como se indica en el código siguiente:

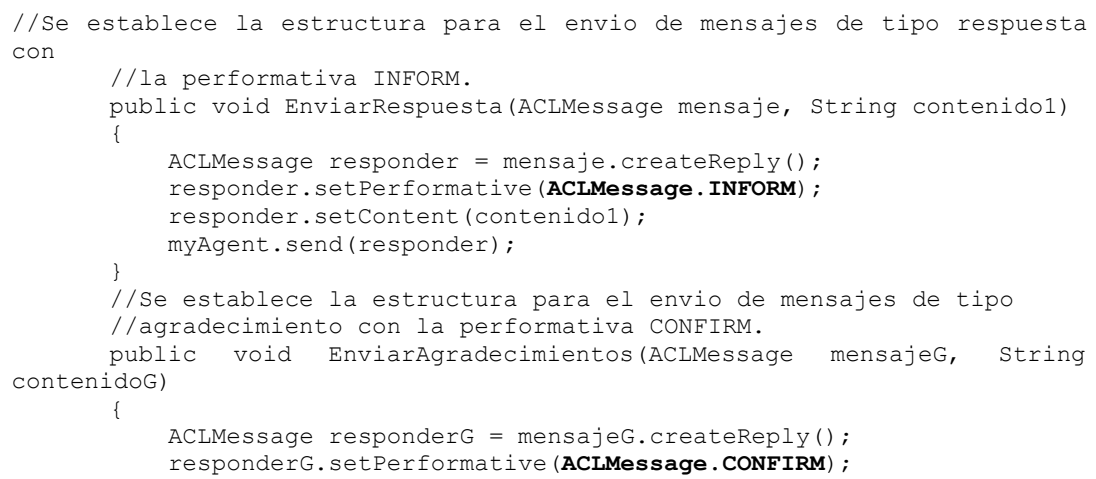




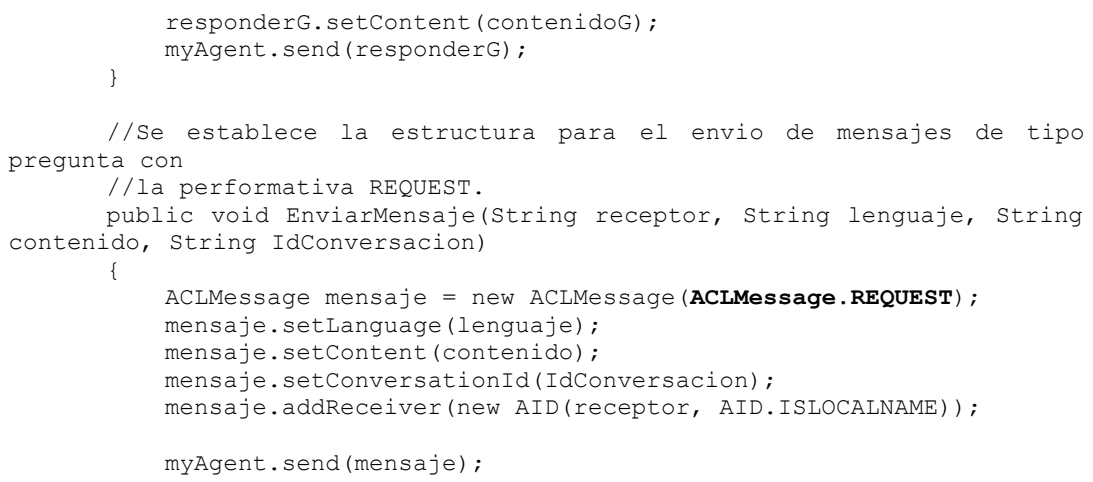

- Inteligencia colectiva al identificar los términos ya clasificados en las entradas. Un ejemplo de esto es cuando el agente Twitter le pregunta al agente Facebook si tiene clasificado el término TB y como éste ya lo tiene clasificado, le responde al agente Twitter que ya lo tiene clasificado, ver Fig. 8.

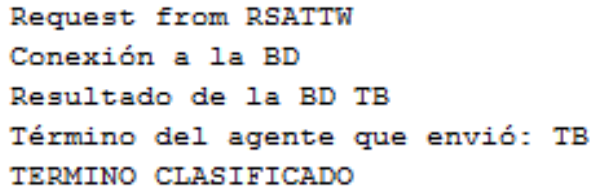

Fig. 8. Ejemplo de un término clasificado por el agente Facebook.

- Planeación en las actividades. Para realizar la clasificación de los términos, las actividades deben seguir un orden; ya que no se puede indicar que un término está clasificado, si antes no se ha almacenado en la BDR SMAterminos. Además, no se puede obtener un término, si no se ha realizado el parseo correspondiente a las entradas de los usuarios.

- Se utilizan Strings que representan contenidos de mensajes (Ontología básica), tal como se muestra en la Fig. 9.

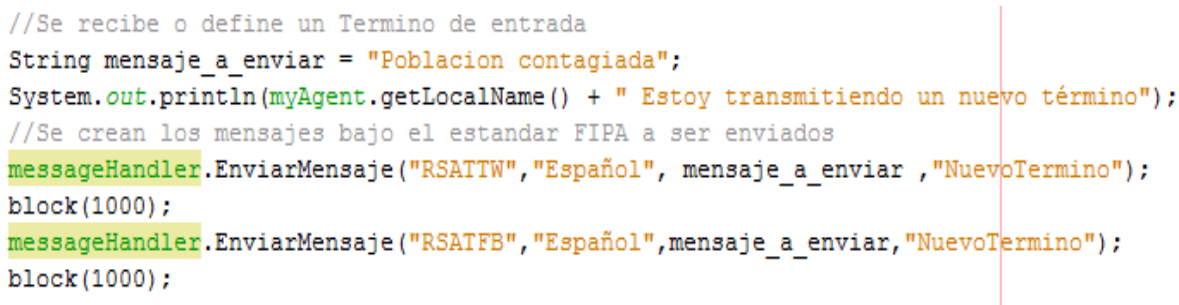

Fig. 9. Ejemplo de código para la propagación de mensajes entre agentes.

- Enriquecer ontologías especializadas con los términos y relaciones previamente identificadas (manualmente); de igual manera, mantener las ontologías actualizadas (manualmente) [3]. Como resultado se tiene el mapeo manual de la BDR "tuberculosis_uno" para obtener la ontología 
Diseño de agentes para recuperar información para el enriquecimiento de ontologías dirigidas ...

actualizada en formato RDF y .ttl para su explotación, Fig. 10 y 11, respectivamente.

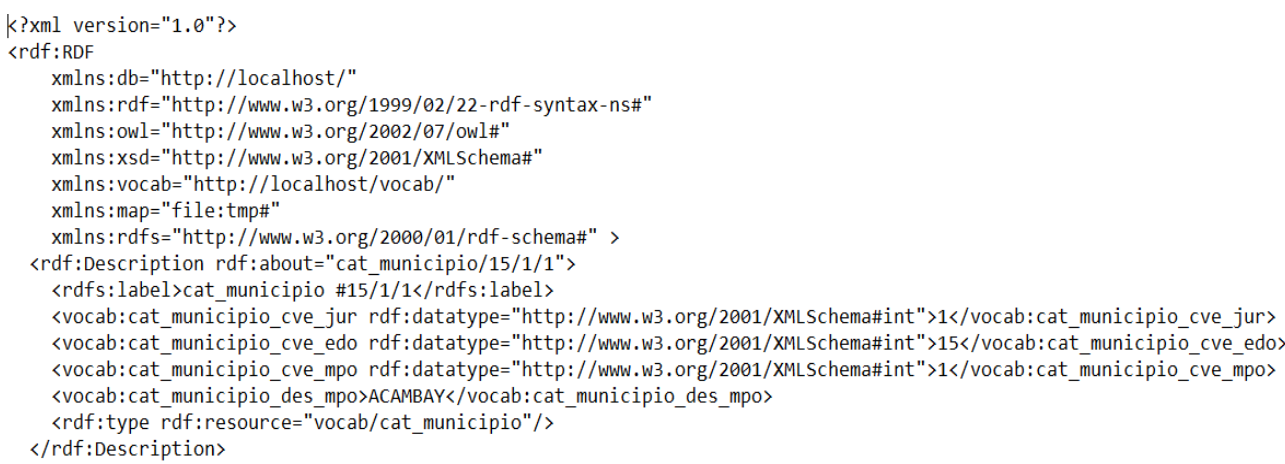

Fig. 10. Ontología obtenida en formato RDF.

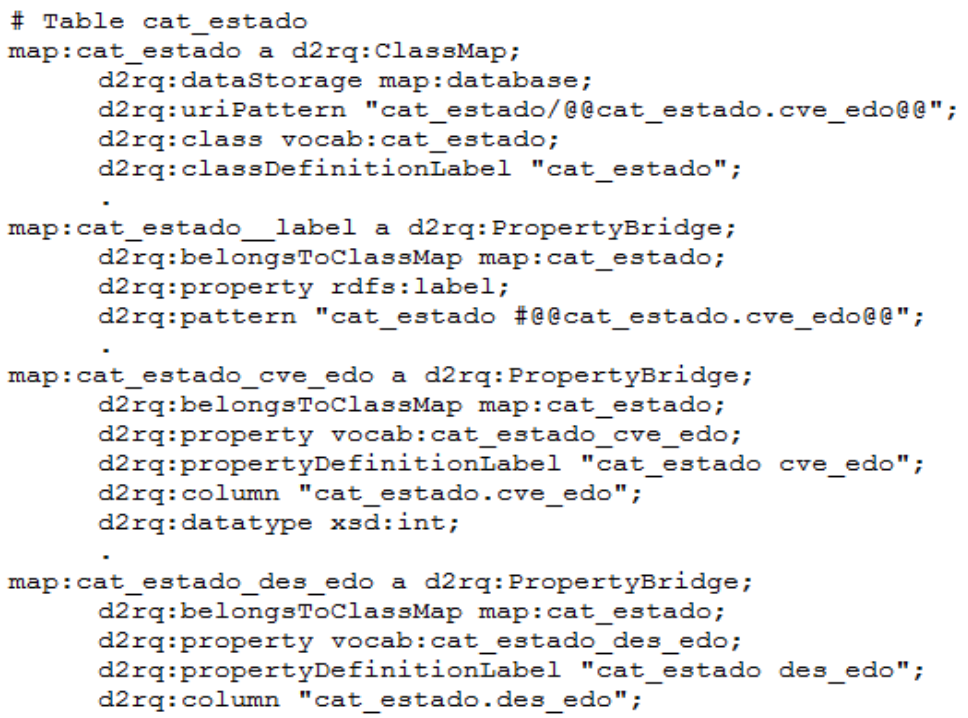

Fig. 11. Ontología obtenida en formato .ttl.

\section{Conclusiones y trabajo futuro}

En este artículo se presenta un Sistema Multiagente que propone un marco (framework) para realizar el enriquecimiento de una ontología al relacionar información proveniente de Bases de Datos relacionales (información estructurada) con información proveniente de la Web (información semiestrucurada). Este marco utiliza la comunicación a través del protocolo FIPA proporcionando un marco común entre los mensajes que deben conocer los agentes para un mejor desempeño en el 
SMA. Proporcionando de esta manera, una herramienta de apoyo a los expertos en el área de Tuberculosis para la recopilación de información en Sitios Web que pudiera ser de relevancia para la actualización de la ontología de Tuberculosis.

Este tipo de propuestas pueden ayudar a extender el conocimiento en áreas que necesitan manejo de datos y experimentación con información diversa para su implementación. El caso de la Epidemiología, y en particular de la Tuberculosis, muestran un campo fértil para poder compaginar todo tipo de información y ayudar a que pequeños focos de infecciones no crezcan demasiado y que la misma sociedad pueda incluso ayudar a combatir las enfermedades.

Como trabajo futuro de esta propuesta, puede concentrarse en la creación de una ontología de uso automática y su actualización cuando ya se tiene implementada en los agentes. De esta manera, se pueden considerar los siguientes puntos:

- La inclusión de un parser que extraiga cada término de las entradas de los Sitios Web considerando la semántica de su contexto.

- La implementación de la ontología de Tuberculosis en los agentes, que se encuentre actualizada con los términos nuevos que se vayan clasificando, cada periodo de tiempo determinado.

- Un agente que se encuentre monitoreando las páginas de Internet que se hayan elegido para la extracción de sus entradas, con la finalidad de verificar la disponibilidad de los mismos en la Web; así como para determinar nuevas páginas que se podrían incorporar para obtener información de acuerdo con la ontología especificada.

\section{Referencias}

1. WORLD WIDE WEB consortium Oficina Española, http://www.w3c.es/Divulgacion/GuiasBreves/WebSemantica\#.

2. Junco R., María de los A. Introducción Sistemas Multiagente. https://sites.google.com/a/itesm.mx/ajunco/home/sma.

3. González, M. D.: Marco Ontológico para la Interoperabilidad en la Explotación de Información en Bases De Datos Heterogéneas. Ph.D. Tesis Doctoral, ITESM Campus Estado de México (2012)

4. Martínez, V. M. de L., González M. M., y Hernández G. N.: Towards a Ubiquitous User Model for Profile Sharing and Reuse. In: Sensors (2012)

5. Julian V., Carrascosa C. y Soler J.: Una arquitectura de sistema multi-agente para la recuperación y presentación de información. Universidad Politécnica de Valencia, Departamento de Sistemas Informáticos y Computación. Valencia (1999)

6. Muñoz N., Cobos C., Rivera W., López J., Mendoza M.: Uso de la metodología GAIA para modelar el comportamiento de personajes en un juego de estrategia en tiempo real. Univ. Antioquia. 53, 214-224 (2010)

7. Junco R., María de los A. Sistemas Multiagente, https://sites.google.com/a/itesm.mx/ajunco/home/sma

8. Jade Tutorial and Primer, http://www.iro.umontreal.ca/ vaucher/Agents/Jade/JadePrimer.html

9. Programación Jade, http://www.programacionjade.wikispaces.com/Agentes\#BookBuyerAgent 
Diseño de agentes para recuperar información para el enriquecimiento de ontologías dirigidas ...

10. Jade Tutorial, Jade Programming for Beginners,

http://jade.tilab.com/doc/tutorials/JADEProgramming-Tutorial-for-beginners.pdf

11. Agents step by step,

https://docs.google.com/presentation/d/1XgzVSKDxcTrYMzMdvvI9IGk501I1uifAQHH0 4BP7zys/edit\#slide=id.i0

12. Barrera M., Núñez H., y Ramos E.: Ingeniería Ontológica, Lecturas en Ciencias de la Computación. Universidad Central de Venezuela, Facultad de Ciencias, Escuela de Computación. Caracas (2012)

13. Sin Tuberculosis, https://twitter.com/SinTuberculosis

14. INSP-Programa de Actualización en Salud Pública y Epidemiología, https://www.facebook.com/INSP.PASPE

15. wordPress.com, http://es.wordpress.com/tag/tuberculosis/ 